44, 320; 1972). Essentially this method gives a soundly-based theoretical expression for the nuclear binding energies, and the fine adjustment of its parameters is made so that it gives the correct results for the heaviest known nuclei.

Brack et al. find that the nucleus with $Z=126$ and $N=228$ is stable against beta decay but has a half life against spontaneous fission of only $39 \mathrm{~ms}$ and an alpha-decay half life of only $18 \mathrm{yr}$. These half lives are extremely sensitive to the calculated binding energies, so that there are uncertainties in these figures of several powers of ten, but even allowing for this it seems that the nucleus is far too unstable to have survived. Major changes in the theories used in these calculations would be required to give sufficiently long half lives.

It thus seems at present that the evidence for superheavy nuclei has been seriously weakened.

\section{Viroids are covalently closed circular RNA}

\section{from Roger Hull}

VIRoIDs are one of the enigmas of plant virology. They are pathogens of higher plants and appear to exist only as self-replicating uncoated ribonucleic acid molecules with many unusual properties.

Much of the knowledge of these properties is based on studies on two viroids, the causal agents of potato spindle tuber (PSTV) and citrus exocortis (CEV) diseases. Using gel electrophoresis and electron microscopy, T. O. Diener and coworkers reported the molecular weight of PSTV to be about $80,000-90,000$ (Virology 53, $70,359 ; 1973)$; J. S. Semancik and coworkers determined a molecular weight of 125,000 for CEV (Virology 53, 448; 1973). These molecular weights are much smaller than those of the RNAs of plant viruses. Thermal melting curves, resistance to nucleases and gel electrophoretic behaviour show that viroids are compact highly structured molecules with a considerable amount of base pairing which makes it difficult to obtain reliable molecular weight estimates using gel electrophoresis. Several models for the molecular structure have been proposed, the most recent suggesting that they are single stranded RNA, base-paired to form hairpin-like shapes (Virology 55, 70; 1973; Virology 63, 160; 1975).

In a recent paper (Proc. natn. Acad. Sci. U.S.A. 73, 3852; 1976) H. L. Sänger et al. describe the results of experiments which lead to a different interpretation of the structure of viroids. They confirmed that viroids were single-stranded molecules by showing that native undenatured nucleic acids had similar molecular weights to denatured molecules. Using sedimentation equilibrium analysis they obtained molecular weight values of 127,000 for PSTV, 119,000 for CEV (from two different hosts) and 110,000 for the viroid causing cucumber pale fruit disease. This confirms the previous estimates of the small size of viroid RNA and shows that they have genetic information to code for a protein no larger than 10,000 daltons. In fact protein synthesis experiments (Virology 61, 486; 1974) indicate that viroid RNA is not translated in vitro.

Sänger et al. went on to determine the structure of viroids. Thermal denaturation showed that each of the viroids they studied had considerable base paining and that there was one relatively long double helical region and not short hairpin sections as in tRNA. Electron microscopy of native nucleic acid revealed that all three viroids have rod-shaped or dumb-bell shaped molecules, as had been previously reported for PSTV (Virology $55,70 ; 1973)$. When viroids which had been treated under strongly denaturing conditions were examined in the electron microscope a variety of structures ranging from rod-like molecules (renatured during the preparation process) to single-stranded circles were found. Sänger and his colleagues then attempted to analyse the nucleotides at the $5^{\prime}$-end and $3^{\prime}$-ends of the viroid molecules. Using standard techniques (phosphorylation of the $5^{\prime}$-end with $5^{\prime}$ polynucleotide kinase and ${ }^{3} \mathrm{H}$-labelling of oxidised 3 '-end and capped 5 '-end by reduction with ${ }^{3} \mathrm{H}$-borohydride) they were unable to detect either $3^{\prime}$ - or $5^{\prime}$ nucleotides. From these and other observations (resistance of viroids to snake venom phosphodiesterase) they concluded that viroids are singlestranded, covalently closed circular RNA molecules.

So far in nature, circular molecules have been found for double-stranded and single-stranded DNA and have been suggested for some doublestranded RNA replicative intermediates (for example, Virology 70, 360; 1976). Viroids therefore appear to be the first examples of a new class of molecule. Sänger et al. point out that they differ from other naturally occurring covalently closed circular molecules in that they are much smaller and that the base pairing is not between complementary strands but within one strand. This structure would aiso explain why viroid RNA is not translated using in vitro systems.

The observations of Sänger et al should help in the current investiga- tions in several laboratories into the origin, mechanisms of replication and pathogenicity of viroids. One major question is whether this new class of molecule occurs normally in uninfected plants; if so, how do they become pathogenic? It is already thought that DNA might be involved at some stage in viroid replication (Virology 64, 106; 1975; Nature 256, $753 ; 1975)$; in fact there are sequence homologies between PSTV RNA and DNA from both infected and healthy plants (Proc. natn. Acad. Sci. U.S.A. $73,2453 ; 1975)$. The search is now on to find circular single-stranded RNA and DNA molecules of viroid size in both infected and healthy plants. I feel that the search should not be restricted to plants. It will be interesting to know how widespread is the occurrence of single-stranded circular RNA molecules and whether they are involved in diseases of organisms other than higher plants.

\section{Most leptons come in pairs}

\section{from $W . T$. Toner}

There was a precursor to the discovery of the $\psi$ : muons and electrons were found in such large numbers in protonnucleus collisions that a new interaction or particle had to be invoked to account for them. (Boymond et al. Phys. Rev. Lett. 33, 112; 1974; Appel et al. ibid, 722; News and Views 254, 103; 1975). Many new studies were begun whose results continue to accumulate. Much of the data can be understood in terms of known processes once the $\psi$ (and perhaps a small amount of charm) is included, but not all.

The large effect is small in any absolute sense, being of the order of one part in ten thousand of the production of the strongly interacting particles whose decays constitute the main experimental background to be subtracted.

The most illuminating results concern the production of pairs of oppositely charged muons or electrons. Several groups find that a substantial fraction of the leptons does come in pairs (see for example Kleberg et al. Phys. Rev. Lett. 37, 1451; 1976) and that the muons are unpolarised as would be the case for electromagnetic production (Leipuner et al. Phys. Rev. Lett. 36, 1011; 1976), although a Russian group has reported a large negative polarisation which is difficult to understand.

The Chicago-Princeton group (K. J. Anderson et al. Phys. Rev. Lett. 37, 799 and 803 ; 1976; Branson et al. to be published) have measured muon pairs 